\title{
REVIEW
}

\section{Status of standard data on shielding calculation in standard committee of the Atomic Energy Society of Japan}

\author{
Yukio Sakamoto $^{\mathrm{a}^{*}}$, Hideo Hirayama ${ }^{\mathrm{b}}$ and the members of the Subcommittee \\ ${ }^{a}$ ATOX Co., Ltd.,1201 Takata, Kashiwa-shi, Chiba, 277-0861, Japan; ${ }^{b}$ High Energy Accelerator Research Organization, 1-1 Oho, \\ Tsukuba, Ibaraki 305-0801, Japan
}

\begin{abstract}
The two standard data on shielding calculation was published or has been prepared by the Standard Committee of the Atomic Energy Society of Japan. One provides dose conversion coefficients from fluence rate to effective dose rate of photons and neutrons for use in radiation shielding calculations. The other provides gamma-ray buildup factors for use in gamma-ray shielding calculations.
\end{abstract}

Keywords: standard data; dose conversion coefficients; effective dose; gamma-ray buildup factors; Invariant Embedding method; Geometrical-Progression fitting formula

\section{Introduction}

In radiation shielding calculations, fluence-to-dose conversion coefficients are necessary to estimate dose rate. This standard gives effective dose conversion coefficients based largely on the ICRP recommendations in 1990. However, original values based on research conducted in Japan have been retained primarily with respect to the extension of energy range and multigroup-wise data.

The gamma-ray buildup factor is defined by the ratio of gamma-ray dose included scattered gamma-ray contribution to dose of unscattered gamma-ray which has no experience of collision from source point to evaluation point. 20 years have passed since publication of standard data by the standard committee of America Nuclear Society at 1991, the extension of data is needed for the evaluation of effective dose and ambient dose equivalent in the shielding calculations, and the codes were also advanced for the evaluation of buildup factors. In the new report, buildup factors obtained by the Invariant Embedding (IE) method and its Geometrical-Progression (GP) fitting formula parameters appeared for 28 materials.

\section{Standard committee in Atomic Energy Society of Japan}

The Radiation Shielding Subcommittee is under Advanced and Fundamental Systems Technical Committee of the Standard Committee in Atomic Energy Society of Japan. Its missions is standardization of methods and data used in shielding calculations, for

*Corresponding author. Email: yukio_sakamoto@atox.co.jp examples, dose conversion coefficients data, gamma-ray buildup factors and typical composition of shielding materials.

The two standard data on shielding calculation was published or has been prepared. One is "Radiation dose conversion coefficients for radiation shielding calculations: 2010" [1]. The other is "Gamma-ray buildup factors: $201 X^{\prime \prime}$.

\section{Dose conversion coefficient standard}

\subsection{Status of dose conversion coefficient data}

The International Commission of Radiation Protection published three reports on the dose conversion coefficients for external radiation in recent 30 years. Publication 51 [2] provided the data of ambient dose equivalent at $10 \mathrm{~mm}$ depth of ICRU phantom. Publication 74 [3] provided the data of effective dose at various irradiation geometries of anthropomorphic phantoms of MIRD-5 model. Publication 116 [4] provided the new data of effective dose of VOXEL phantom.

The standard committee of American Nuclear Society also published two reports on dose conversion coefficients. ANSI/ANS-6.1.1(1977) [5] and ANSI/ANS-6.1.1(1991) [6] provided the energy fitting parameters for maximum dose equivalent in slab phantom and for effective dose equivalents, respectively.

In Japan, the standard data on dose conversion coefficients is needed in the view points of following items, high-energy data over the energy region shown by ICRP reports, group-wise data and energy fitting parameters for effective dose. The standard committee in the Atomic Energy Society of Japan published standard 
data of dose conversion coefficients [1].

\subsection{Basic data on dose conversion coefficients}

Table 1 shows the energy range and irradiation geometries of dose conversion coefficients in various reports. ICRP Publication 74 shows data in 6 geometries in the energy range of $0.01-10 \mathrm{MeV}$ and $1 \mathrm{meV}-180$ $\mathrm{MeV}$, for photons and neutrons, respectively. Japanese domestic laws on radiation protection requires the data of Anterior-Posterior irradiation geometry (irradiation geometry from front side of phantom model to back side) in the energy range of $0.01-10 \mathrm{MeV}$ and $1 \mathrm{meV}-$ $20 \mathrm{MeV}$, for photons and neutrons, respectively. In the shielding calculations in the high energy accelerators, data for high energy photons and neutrons are required. Standard data extend the energy region up to $10 \mathrm{GeV}$ for photons and $5 \mathrm{GeV}$ for neutrons, respectively. Standard data include the data at specific energy and corresponding to group averaged, and fitting parameters of polynomial expressions.

Table 1. Energy range and irradiation geometries of dose conversion coefficients in various reports.

\begin{tabular}{|c|l|l|}
\hline & Photons & Neutrons \\
\hline ICRP74 & $0.01-10 \mathrm{MeV}$ & $1 \mathrm{meV}-180 \mathrm{MeV}$ \\
& 6 Geometries & $6 \mathrm{Geometries}$ \\
\hline Domestic & $0.01-10 \mathrm{MeV}$ & $1 \mathrm{meV}-20 \mathrm{MeV}$ \\
Law(Japan) & AP Geometry & AP Geometry \\
\hline High Energy & EGS4: $1 \mathrm{MeV}-10 \mathrm{GeV}$ & HETC-3STEP: \\
Data & FLUKA: & $20 \mathrm{MeV}-10 \mathrm{GeV}$ \\
& $0.05 \mathrm{MeV}-100 \mathrm{GeV}$ & FLUKA: \\
& & $1 \mathrm{meV}-100 \mathrm{GeV}$ \\
\hline Scope & $0.01-10 \mathrm{GeV}$ & $1 \mathrm{meV}-5 \mathrm{GeV}$ \\
& AP Geometry & AP Geometry \\
\hline
\end{tabular}

\subsection{Data for photons}

Figure 1 shows the data for AP irradiation of high-energy photons. In the high-energy region, the transportation of produced charge particles affects dose conversion coefficients. ICRP Publication 74 adopted Kerma approximation where the produced electrons deposit the energy within near region of produced position. The adoption of Kerma approximation for high energy photos reveals the overestimation of dose conversion coefficients because electrons have large range.

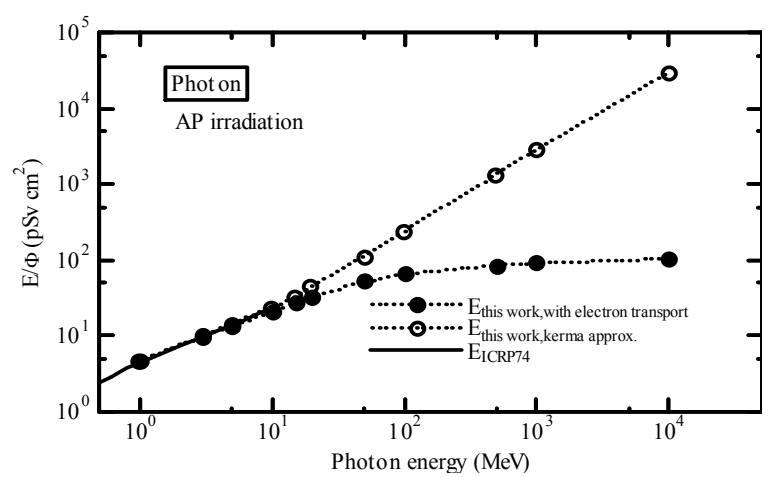

Figure 1. Effective dose at AP irradiation of high-energy photons.

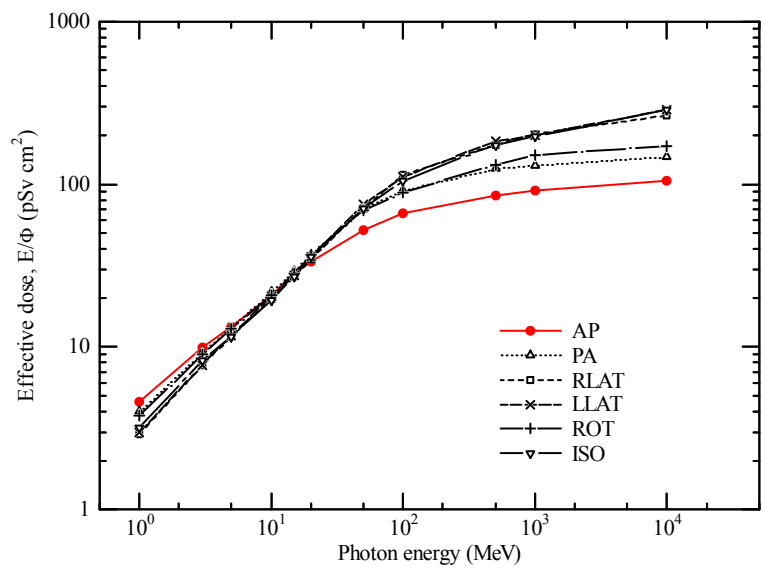

Figure 2. Effective dose at various irradiation geometries of high-energy photons.

Figure 2 shows the data of effective dose at various irradiation geometries for high-energy photons. Above few $\mathrm{MeV}$, data for AP irradiation geometry is not maximum value of effective dose. But data for AP irradiation geometry were adopted in the standard data in the view point of the consistency to the low energy region where domestic law adopted the data for AP irradiation geometry.

\subsection{Data for neutrons}

Figure 3 shows radiation weighting factor shown by ICRP Publication 60 and the average quality factors obtained by the calculations of effective dose equivalents for high-energy neutrons. The value of 5 for radiation weighting factor of high-energy neutrons is conservative compared to average quality factors of three irradiation geometries for high-energy neutrons.

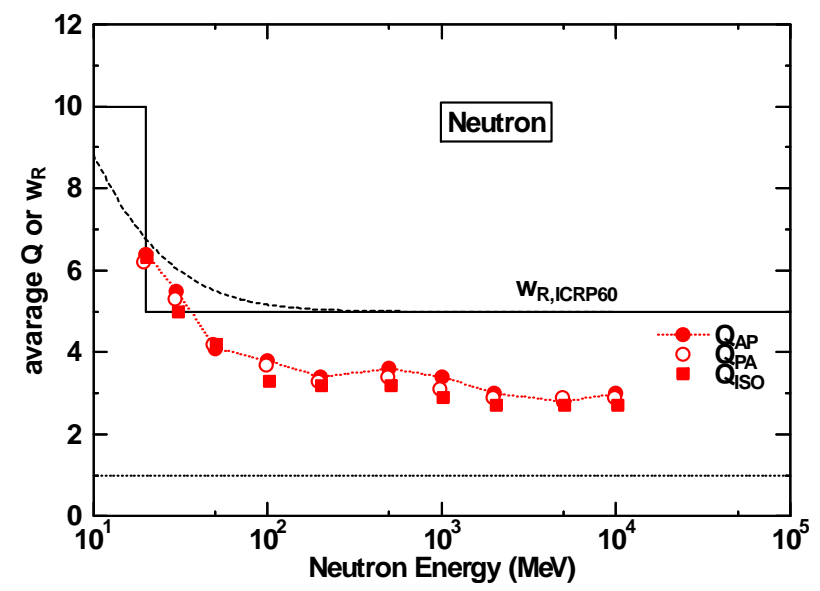

Figure 3. Radiation weighting factor and average of quality factors for high-energy neutrons.

Figure 4 shows the data of effective dose at various irradiation geometries for high-energy neutrons. The data for AP irradiation geometry were also adopted in the standard data by the same reason in the case of photons. The degree of underestimation was studied by 
using the concrete transmitted neutron spectra produced in iron targets by the irradiation of $0.6,1$ and $1.5 \mathrm{GeV}$ protons and emitted to 90 degree direction. The difference of total effective dose between dose conversion coefficient at AP irradiation geometry and the maximum dose conversion coefficient is below about $20 \%$ [7].

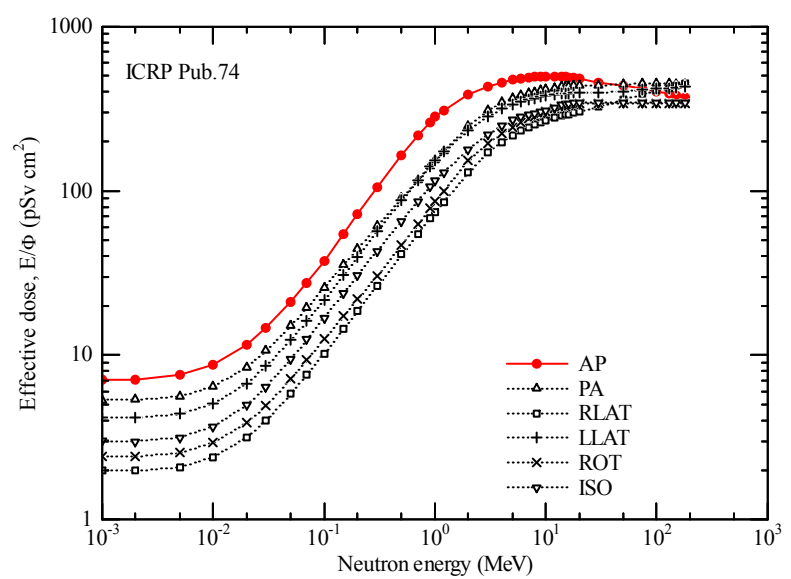

Figure 4. Effective dose at AP irradiation of high-energy neutrons.

\subsection{Fitting formula expressed by polynomial function}

For effective dose conversion coefficient at desired energy point, the following fitting formula expressed by polynomial function is shown in standard report for photons and neutron, respectively.

$$
\begin{aligned}
& {[\mathrm{E} / \Phi]_{\text {photon }}=\exp \left\{\sum_{\mathrm{m}=0}^{4} \mathrm{M}_{\gamma}{ }^{\mathrm{m}} \times\left(\ln \mathrm{E}_{\gamma}\right)^{\mathrm{m}}\right\}} \\
& {[E / \Phi]_{\text {neuron }}=\exp \left\{\sum_{m=0}^{5} M_{n}{ }^{m} \times\left(\ln E_{n}\right)^{m}\right\}}
\end{aligned}
$$

Fitting errors for photons are below $3 \%$, and those for neutrons are below $6 \%$ except $100 \mathrm{MeV}$ data.

\subsection{Group averaged data for typical shielding group constants}

Table 2. Group averaged effective dose and ambient dose equivalent for energy group structure.

\begin{tabular}{|l|c|c|}
\hline $\begin{array}{l}\text { Energy Group } \\
\text { Structure (n/g) }\end{array}$ & Effective dose & $\begin{array}{c}\text { Ambient Dose } \\
\text { Equivalent }\end{array}$ \\
\hline $\begin{array}{l}\text { DLC23/CASK } \\
(22 \mathrm{n} / 18 \mathrm{~g})\end{array}$ & 0 & $\circ$ \\
\hline BUGL-96 (47n/20g) & 0 & 0 \\
\hline JSD-J2 (100n/20g) & $\circ$ & 0 \\
\hline $\begin{array}{l}\text { VITAMIN-B6 } \\
(199 \mathrm{n} / 42 \mathrm{~g})\end{array}$ & 0 & 0 \\
\hline $\begin{array}{l}\text { High Energy } \\
(78 \mathrm{n} / 22 \mathrm{~g} \text { Extended } \\
\text { HILO86 Up to 3GeV) }\end{array}$ & 0 & - \\
\hline
\end{tabular}

Group average effective dose and ambient dose equivalent for 5 energy group structures, DLC23/CASK (22 neutron and 18 gamma-ray groups), BUGL-96 (47n, 20g), JSD-J2 (100n, 20g), VITAMIN-B6 (199n, 42g) and high energy group constant $(78 \mathrm{n}, 22 \mathrm{~g})$, were prepared as standard data shown in Table 2.

\section{Gamma-ray buildup factor standard}

\subsection{Status of gamma-ray buildup factors}

The gamma-ray buildup factors of ANSI/ANS-6.4.3 (1991) [8] are widely used in the shielding calculation for RI gamma-ray sources. This standard includes the Japanese contributions, which are buildup factors for high-atomic-number materials evaluated by PALLAS code with consideration of Bremsstrahlung and fluorescence, data checking for low-atomic-number materials and GP fitting parameters.

Over 20 yeas passed after publication of ANSI/ANS-6.4.3, we have the motivation of revision of gamma-ray buildup factors in the following reasons, 1) data produced by same evaluation code and same cross section data, 2) data corresponding to deep penetration up to $100 \mathrm{mfp}$ of shielding thickness, 3) introduction of more accurate Bremsstrahlung matrix, 4) data of effective dose for AP and ISO irradiation geometries and ambient dose equivalent, and 5) revision of GP formula.

\subsection{Comparison of buildup factor standard between ANS and AESJ}

The data in AESJ standard were prepared by the IE method coupled with Bremsstrahlung matrix calculated by EGS4 code. ANS data were prepared for low-Z materials by the moments method and for high- $Z$ materials by PALLAS code. Table 3 shows the comparison of gamma-ray buildup factors between ANS and AESJ standard. The data of effective dose for AP and PA irradiation geometries, and ambient dose equivalent were added to those for exposure and absorbed dose in AESJ standard. The data for rubidium (atomic number Z: 37) and bismuth (Z: 83) were added to those of 23 elements from beryllium to uranium, and water, concrete and air. Energy range of gamma-ray is from 0.015 to $15 \mathrm{MeV}$, same as ANS standard. The thickness of shielding material is extended to $100 \mathrm{mfp}$. The treatment of scattering and fluorescence in photon transport is same as that in ANS standard. The contribution of Bremsstrahlung is considered in all materials, and the production matrix of Bremsstarlung is prepared with gamma shower code EGS4. In ANS standard, the contribution of Bremsstrahlung was limited to high-Z materials from molybdenum to uranium, and the production of Bremsstrahlung is approximated by forward direction emission. Photon cross section and energy absorption coefficients in IE method were based on PHOTX.

Gamma-ray buildup factor data obtained by IE method were evaluated by the comparison with 
moments method data in ANS standard and EGS4 results, and checked by sensitivity analyses to spatial mesh, angular mesh and number of energy group.

Table 3. Comparison of gamma-ray buildup factors between ANS and AESJ standard.

\begin{tabular}{|c|c|c|}
\hline Item & ANSI/ANS-6.4.3(1991) & $\begin{array}{l}\text { AESJ-SC } \\
-A 00 X: 201 X\end{array}$ \\
\hline Dose & Exposure, Absorbed Dose & $\begin{array}{l}\text { Added } \quad \text { E(AP) } \\
\text { E(ISO), } \mathrm{H}^{*}(10)\end{array}$ \\
\hline Material & $\begin{array}{l}\text { Be-U ( } 23 \text { elements), water, } \\
\text { concrete, air }\end{array}$ & Added $\mathrm{Rb}$ and $\mathrm{Bi}$ \\
\hline Energy & $0.015-15 \mathrm{MeV}$ & same \\
\hline Thickness & $0.5-40 \mathrm{mfp}$ (16 points) & $\begin{array}{l}0.5-100 \mathrm{mfp}(2 s \\
\text { points })\end{array}$ \\
\hline & $\begin{array}{l}\text { Compton Scattering by } \\
\text { Free Electron }\end{array}$ & Same \\
\hline Fluore & With consideration & Same \\
\hline Bremsstarlung & $\begin{array}{l}\text { Only high-Z Materials } \\
\text { (forward emission) }\end{array}$ & $\begin{array}{l}\text { All Materials } \\
\text { (EGS4 Results) }\end{array}$ \\
\hline $\begin{array}{c}\text { X-sec } \\
\text { Energy } \\
\text { Absorption }\end{array}$ & $\begin{array}{l}\text { NBS29 / PHOTX } \\
\text { NISTIR } 5632\end{array}$ & $\begin{array}{l}\text { PHOTX } \\
\text { Same }\end{array}$ \\
\hline Code & Moments / PALLAS & IE Method \\
\hline Fitting Formula & $\begin{array}{l}\text { GP Methods and Taylor's } \\
\text { Formula }\end{array}$ & $\begin{array}{l}\text { Revised } \\
\text { Method }\end{array}$ \\
\hline
\end{tabular}

\subsection{Fitting function to gamma-ray buildup factors}

In the shielding calculation of gamma-ray source by point kernel code, the buildup factors at desired shielding thickness were required. For such purposes, fitting function of Geometrical-Progression (GP) formula is prepared in AESJ standard. The gamma-ray buildup factors at $X \mathrm{mfp}$ of shielding thickness is obtained by Eq. (3) and (4) for $K \neq 1$ and $K=1$, respectively, where parameters $b$ and $K$ are corresponding to a buildup factor at $1 \mathrm{mfp}$ and a multiplication factor of dose through $1 \mathrm{mfp}$ photon penetration, respectively. Parameter $K$ is obtained by Eq. (5) for $X$ below $X_{\mathrm{m}}$ using parameters $c, a, d$ and $X_{\mathrm{k}}$. For $X$ above $X_{\mathrm{m}}, K$ is obtained by Eqs. (6) and (7), where $K_{\mathrm{m}}$ and $K_{\mathrm{m}-1}$ are parameters $K$ at $X_{\mathrm{m}}$ and $X_{\mathrm{m}-1}$, respectively. In former formula, $\xi$ was fixed to 0.1 .

$$
\begin{aligned}
& B(E, X)=1+(b-1) \frac{K^{X}-1}{K-1} \quad \text { for } K \neq 1 \\
& B(E, X)=1+(b-1) X \quad \text { for } K=1 \\
& K(E, X)=c X^{a}+d \frac{\tanh \left(X / X_{k}-2\right)-\tanh (-2)}{1-\tanh (-2)} \\
& \text { for } 0 \leqq X \leqq X_{\mathrm{m}} \text { and } X_{\mathrm{m}}=40 \mathrm{mfp}
\end{aligned}
$$

$$
\begin{aligned}
& K(E, X)=1+\left(K_{m-1}-1\right)\left[\frac{K_{m}-1}{K_{m-1}-1}\right]^{\xi(X)} \\
& \text { for } X>X_{\mathrm{m}} \text { and } X_{\mathrm{m}}<100 \mathrm{mfp}
\end{aligned}
$$

where

$$
\xi(X)=\frac{\left(X / X_{m-1}\right)^{\eta}-1}{\left(X_{m} / X_{m-1}\right)^{0.1}-1}
$$

The detail of GP fittings to JAES standard is written in Harima's paper of this proceedings.

\section{Conclusion}

Dose conversion coefficients standard gives data to obtain effective dose for photons having energies up to $10 \mathrm{GeV}$, where secondary electron transport is taken into consideration in the energy range above $5 \mathrm{MeV}$. Also the dose conversion coefficients standard to obtain effective dose for neutrons having energies up to $5 \mathrm{GeV}$ are given in preparation for construction planning of middle and high energy proton accelerators in the near future.

In the new report of gamma-ray buildup factors, data obtained by the IE method and its GP fitting formula parameters appeared for 28 materials, with the energy range from $0.015 \mathrm{MeV}$ to $15 \mathrm{MeV}$, up to $100 \mathrm{mfp}, 5$ dose quantities of exposure, effective dose with AP and ISO irradiation geometries, absorbed dose and ambient dose equivalents.

In the future plan, standard data of dose conversion coefficient will adopt ICRP Pub.116 data. After public comments, gamma-ray buildup factor standard will be published, and introduced into point kernel code. English version will be published near future. There is another plan to make up the standard of material compositions for shielding calculations and activation analyses.

\section{References}

[1] Standard Committee of Atomic Energy Society of Japan, "Radiation dose conversion coefficients for radiation shielding calculations: 2010", AESJ-SC-R002.2010. [in Japanese]

[2] International Commission on Radiological Protection, "Data for Use in Protection against External Radiation: ICRP Publication 51,"Annals of the ICRP, 17(2-3) (Pergamon Press) (1987).

[3] International Commission on Radiological Protection, "Conversion Coefficients for Use in Radiological Protection Against External Radiation: ICRP Publication 74," Annals of the ICRP, 26(3/4) (Oxford: Elsevier Science) (1996).

[4] International Commission on Radiological Protection, "Conversion Coefficients for Radiological Protection Quantities for External 
Radiation Exposures: ICRP Publication 116," Annals of the ICRP, 40(2/5) (Oxford: Elsevier Science) (2012).

[5] American National Standard, Neutron and Gamma-Ray Flux-to-Dose Rate Factors, ANSI/ANS-6.1.1-1977 (1977).

[6] American National Standard, Neutron and Gamma-Ray Flux-to-Dose Rate Factors, ANSI/ANS-6.1.1-1991 (1991).

[7] Y. Sakamoto and Y. Yamaguchi, Dose Conversion
Coefficients in the Shielding Design Calculation for High Energy Proton Accelerator Facilities, JAERI-Tech 2001-042, Japan Atomic Energy Research Institute,(2001).

[8] American National Standard, Gamma-Ray Attenuation Coefficients and Buildup Factors for Engineering Materials, ANSI/ANS-6.4.3-1991 (1991). 